\title{
On the Bowen fluorescence mechanism in the helium-oxygen plasmas
}

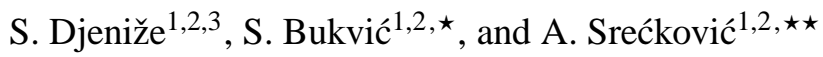 \\ ${ }^{1}$ Faculty of Physics, University of Belgrade, POB 368, Belgrade, Serbia \\ 2 Isaac Newton Institute of Chile, Yugoslavia Branch, Belgrade, Serbia \\ ${ }^{3}$ Hungarian Academy of Sciences, Budapest, Hungary
}

Received 19 June 2003 / Accepted 8 August 2003

\begin{abstract}
The dependence of the radiation intensity from the $2 \mathrm{p} 3 \mathrm{~d}{ }^{3} \mathrm{P}_{2,1,0}^{\mathrm{o}}$ doubly ionized oxygen (O III) levels on the He/O density ratio has been investigated in optically thin laboratory plasmas. A clear contribution of the astrophysically important Bowen mechanism to the most intensive $313.279 \mathrm{~nm}$ O III line radiation has been found in the primary O III Bowen cascade. We have found that in plasmas with electron temperatures of about $50000 \mathrm{~K}$ and electron densities higher than $10^{22} \mathrm{~m}^{-3}$, the $312.163 \mathrm{~nm}$ O III spectral line also shows a fluorescence tendency caused by the Bowen mechanism. On the basis of the established dependence of the fluorescence efficiency on the He/O density ratio we recommend the $I(313.279 \mathrm{~nm}) / I(311.567 \mathrm{~nm})$ and $I(312.163 \mathrm{~nm}) / I(311.567 \mathrm{~nm}) \mathrm{O}$ III line intensity ratios as a measure of the presence of the helium/oxygen density ratio in astrophysical plasmas. The line intensity ratio related to the $344.405 \mathrm{~nm}$ and $342.863 \mathrm{~nm}$ O III lines (which also belong to the primary cascade in the Bowen fluorescence mechanism and originate in the same energy level) has also been monitored in pure oxygen and helium-oxygen plasmas. We have found a good agreement with the results of previous astrophysical observations and recently published theoretical predictions. We have also found that the $I(344.405 \mathrm{~nm}) / I(342.863 \mathrm{~nm})$ line intensity ratio does not depends on the helium presence in plasmas and thus, it represents a convenient value in plasma spectroscopy.
\end{abstract}

Key words. plasmas - atomic data

\section{Introduction}

In the Bowen mechanism (Bowen 1934; Kallman \& McCray 1980; Saraph \& Seaton 1980) the $2 \mathrm{p} 3 \mathrm{~d}^{3} \mathrm{P}_{2}^{\mathrm{o}}$ doubly ionized oxygen (O III) level (with $40.85 \mathrm{eV}$ excitation energy) is populated through pumping by the ultraviolet $\mathrm{He}$ II $\mathrm{Ly}_{\alpha}$ resonance line at $30.378 \mathrm{~nm}$. The excited state then decays through a series of primary, secondary and tertiary cascades (Figs. 1 and 2 in Saraph \& Seaton 1980 and Fig. 1 in Kallman \& McCray 1980). Some of these lines have been observed in optical spectra of nebulae (Aller et al. 1966; Liu \& Danziger 1993; Shrader et al. 1997) and others have been observed with the International Ultraviolet Explorer (IUE) satellite (Harrington et al. 1981). It is to be expected that these O III lines have higher intensities than the population processes provide, based on the LTE model, especially in the case of the $313.279 \mathrm{~nm}$, $344.405 \mathrm{~nm}$ and $342.863 \mathrm{~nm}$ lines in the primary cascade which originates in the pumped $2 \mathrm{p} 3 \mathrm{~d}{ }^{3} \mathrm{P}_{2}^{\mathrm{o}}$ level. The intensity of the fluorescence must depend on the density of the He II Ly $\mathrm{L}_{\alpha}$ photons. Therefore, plasma composition and conditions (i.e. electron temperature $(T)$ and density $(N)$ ) clearly determine

Send offprint requests to: S. Djeniže, e-mail: steva@ff.bg.ac . yu

* e-mail: ebukvic@ff.bg.ac.yu

$\star \star$ e-mail: srecko@ff.bg.ac.yu

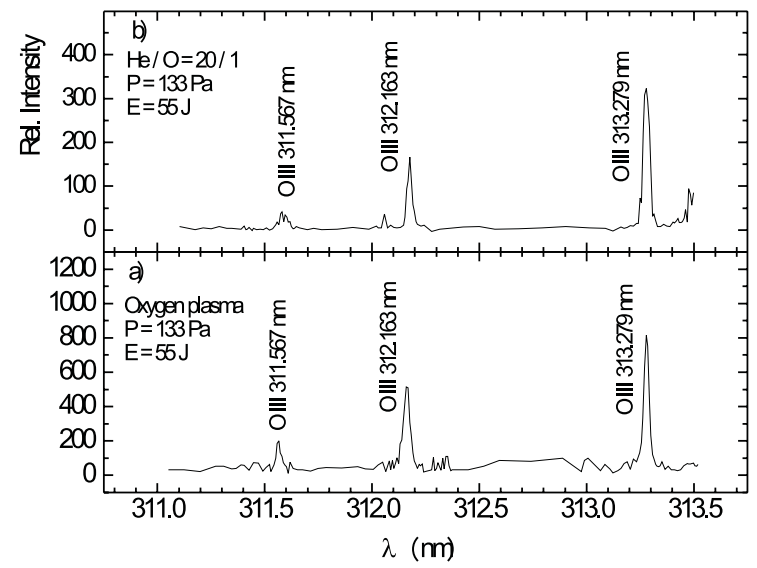

Fig. 1. Recorded O III lines at various plasma compositions recorded at 5 th $\mu \mathrm{s}$ a) and 10 th $\mu \mathrm{s} \mathrm{b}$ ) after the beginning of the discharge. $P$ and $E$ denote discharge pressure and bank energy, respectively.

this mechanism. As a measure of the presence and efficiency of the Bowen fluorescence mechanism, spectral line intensity ratios within O III spectral lines could be used. One of them comes from the Bowen cascades and the second is out of the cascades. 
The aim of this work is to present, for the first time, the dependence of the efficiency of the Bowen fluorescence mechanism in the $\mathrm{O}$ III spectrum on the $\mathrm{He} / \mathrm{O}$ abundance ratio and plasma parameters ( $T$ and $N$ ) on the basis of the measured O III line intensity ratios. As a pilot line, we have chosen the most intensive $313.279 \mathrm{~nm}$ O III line origins from the pumped $2 \mathrm{p} 3 \mathrm{~d}^{3} \mathrm{P}_{2}^{\mathrm{o}} \mathrm{O}$ III level (with $40.85 \mathrm{eV}$ excitation energy) for which the Bowen mechanism has shown and confirmed. The $312.163 \mathrm{~nm}$ (from the $2 \mathrm{p} 3 \mathrm{~d}{ }^{3} \mathrm{P}_{1}^{\mathrm{o}}$ level with $40.86 \mathrm{eV}$ energy) and $311.567 \mathrm{~nm}$ (from the $2 \mathrm{p}^{1} \mathrm{~d}^{3} \mathrm{P}_{0}^{\mathrm{o}}$ level with $40.87 \mathrm{eV}$ energy) lines which come from the mutually very close energy levels in the same transition have also been included in investigation. Also, we have monitored the spectral line intensity ratio related to the $344.405 \mathrm{~nm}$ and $342.863 \mathrm{~nm}$ O III lines which also belong to the primary Bowen cascade (both arise from the $2 \mathrm{p} 3 \mathrm{~d}^{3} \mathrm{P}_{2}^{\mathrm{o}}$ level) in pure oxygen plasma and also in heliumoxygen plasmas. Their existing experimental (Aller et al. 1966; Liu \& Danziger 1993) and theoretical (Saraph \& Seaton 1980; NIST 2003, and references therein) values show high internal scatter up to a factor of 2 .

\section{Experiment}

A modified version of the linear low pressure pulsed arc (Djeniže \& Bukvić 2001; Djeniže et al. 2002a-c; Milosavljević \& Djeniže 2002) has been used as an optically thin plasma source. A pulsed discharge was performed in a pyrex discharge tube (with quartz windows) of $5 \mathrm{~mm}$ inner diameter and plasma length of $14 \mathrm{~cm}$. The working gases were pure oxygen and various helium-oxygen mixtures (He/O: 0.9, 5 and 20 ) at $133 \mathrm{~Pa}$ pressure in flowing regime. The $\mathrm{He} / \mathrm{O}$ density ratio includes neutrals and all other ionization stages. The capacitor of $14 \mu \mathrm{F}$ was charged up to $2.8 \mathrm{kV}$. The complete line profile and intensity $(I)$ recording procedures together with the experimental set-up used have been described in Djeniže et al. 2002a,b, 2003). As an example, the recorded $313.279,312.163$ and $311.567 \mathrm{~nm}$ O III line profiles are presented in Fig. 1 for two different plasmas.

The electron temperature was determined from the ratios of the relative intensities (Saha equation) of O III $(326.08 \mathrm{~nm}$, $326.53 \mathrm{~nm}$ and $326.72 \mathrm{~nm}$ ) and O II (327.08 $\mathrm{nm}$ and $327.34 \mathrm{~nm})$ spectral lines with an estimated error between $\pm 8 \%$ and $\pm 12 \%$, assuming the existence of LTE, according to the criterion from Griem (1964) and Rompe \& Steenbeck (1967). The mentioned $\mathrm{O}$ III lines are out of the Bowen cascades and their parent energy levels (with about $40.25 \mathrm{eV}$ excitation energy) are populated according to the predictions made by the LTE model. In our experiments, the electron densities are about $5 \times 10^{22} \mathrm{~m}^{-3}$ (in the 10th $\mu \mathrm{s}$ after the beginning of the discharge; see Fig. 2 in Djeniže et al. 2003) satisfying the criterion for the existence of the LTE. This criterion (Rompe \& Steenbeck 1967) support the LTE at $N>2.4 \times 10^{22} \mathrm{~m}^{-3}$ (at $50000 \mathrm{~K}$ electron temperature) for the $\mathrm{O}$ III lines used. The necessary atomic data are taken from NIST (2003). The obtained electron temperatures were about $50000 \mathrm{~K}$ (within $\pm 8 \%$ ) in the case of heliumoxygen plasmas. The electron density decay was measured using the well-known single laser interferometry technique for the $632.8 \mathrm{~nm} \mathrm{He}-\mathrm{Ne}$ laser wavelength with an estimated error
Table 1. Characteristics of the investigated O III transitions. $J_{f}$ and $J_{i}$ are the inner quantum numbers of the final $(f)$ and initial $(i)$ state of the transition. $E_{i}$ and $g_{i}$ represent initial energy levels and their statistical weights. Atomic data are taken from NIST (2003).

\begin{tabular}{|c|c|c|c|}
\hline \hline $\begin{array}{c}\text { Multiplet } \\
J_{f}-J_{i}\end{array}$ & $\begin{array}{c}\lambda \\
(\mathrm{nm})\end{array}$ & $\begin{array}{c}E_{i} \\
(\mathrm{eV})\end{array}$ & $g_{i}$ \\
\hline $3 \mathrm{p}^{3} \mathrm{~S}-3 \mathrm{~d}^{3} \mathrm{P}^{\mathrm{o}}$ & & & \\
$1-2$ & 313.279 & 40.85 & 5 \\
$1-1$ & 312.163 & 40.86 & 3 \\
$1-0$ & 311.567 & 40.87 & 1 \\
$3 \mathrm{p}^{3} \mathrm{P}-3 \mathrm{~d}^{3} \mathrm{P}^{\mathrm{o}}$ & & & \\
$1-2$ & 342.863 & 40.85 & 5 \\
$2-2$ & 344.405 & 40.85 & 5 \\
\hline
\end{tabular}

of $\pm 6 \%$. In the case of helium-oxygen plasmas they are higher than $10^{22} \mathrm{~m}^{-3}$ from the 4 th $\mu$ s up to the 45 th $\mu$ s after the beginning of the discharge when the line intensity ratios have been monitored. We have monitored the chosen O III spectral line intensities during the whole plasma decay period together with the He II $\mathrm{P} \alpha(468.6 \mathrm{~nm})$ spectral line intensity. The moment of realization of the $\mathrm{He}$ II $\mathrm{P} \alpha$ line intensity maximum is also the moment when the concentration of the He II ions is at its maximum. We expect that the presence of the $30.378 \mathrm{~nm}$ He II resonance photon density, essential for the Bowen fluorescence mechanism, also has its maximum at this moment. At this moment the efficiency of the Bowen fluorescence must have its maximum. We expect that this efficiency depends on the $\mathrm{He} / \mathrm{O}$ density ratio.

\section{Line intensity ratio}

When two spectral lines (index 1 and 2) arise from the mutually very close upper energy levels (see Table 1) their relative line intensity ratio (practically independent of the electron temperature) is given as (Griem 1964):

$I_{1} / I_{2}=A_{1} g_{1} \lambda_{2} / A_{2} g_{2} \lambda_{1}$

where $A, g$ and $\lambda$ denote transition probability, statistical weight and the wavelength of the transitions. $g_{1}=g_{2}$ for the same parent energy levels. The knowledge of these atomic data (NIST 2003) enables the comparison between the experimental and theoretical line intensity ratios. The characteristics of the investigated O III transitions are presented in Table 1.

\section{Results}

Our measured O III line intensity ratios during the whole plasma decay interval are presented in Fig. 2 for various plasma compositions together with the normalized He II $468.6 \mathrm{~nm}$ line intensity decays.

Our line intensity ratios related to the $344.405 \mathrm{~nm}$ and $342.863 \mathrm{~nm}$ O III lines are presented in Table 2 together with the results of other authors. 




Fig. 2. O III line intensity ratios during the plasma decay in various a)-d) plasma compositions. Symbols $\bullet, \Delta$ and $\circ$ correspond to the $I(313.3) / I(311.6), I(312.2) / I(311.6)$ and $I(313.3) / I(312.2)$ O III line intensity ratios, respectively. Full lines represent line intensity ratios calculated on the basis of Eq. (1) using transition probabilities tabulated in NIST (2003). Dashed lines denote normalized experimental line intensity maximum of the He II P $\alpha(468.6 \mathrm{~nm})$ spectral line. Electron temperature $(T)$ is related to the 10 th $\mu$ s after the beginning of discharge when the $\mathrm{P} \alpha$ line has its intensity maximum and the Bowen mechanism comes up to its maximum. Error bar represents estimated uncertainties of $\pm 8 \%$.

Table 2. The $I(344.4) / I(342.9)$ O III line intensity ratios. Symbols: $T w(\mathrm{O}), T w(\mathrm{He} / \mathrm{O}=0.9)$ denote our values obtained in oxygen and heliumoxygen mixture within $\pm 8 \%$ uncertainties. Results from other data sources are given with: A (Aller et al. 1966); LD (Liu \& Danziger 1993); FF (Froese Fischer 1994); AG (Aggarwal et al. 1997); KB (Kastner \& Bhatia 1990); BK (Bhatia \& Kastner 1993); SS (Saraph \& Seaton 1980); N (NIST 2003).

\begin{tabular}{cccccccccc}
\hline \hline$T w(\mathrm{O})$ & $T w(\mathrm{He} / \mathrm{O})$ & $\mathrm{A}$ & $\mathrm{LD}$ & $\mathrm{FF}$ & $\mathrm{AG}$ & $\mathrm{KB}$ & $\mathrm{BK}$ & $\mathrm{SS}$ & $\mathrm{N}$ \\
\hline 6.50 & 5.95 & 6.40 & 5.40 & 6.53 & 5.23 & 6.10 & 5.52 & 2.98 & 2.95 \\
\hline
\end{tabular}

\section{Discussion}

\subsection{Oxygen plasma}

In case of pure oxygen plasma we have found line intensity ratios (see Fig. 2a) which agree very well (within $8 \%$ experimental accuracy) with the theoretical ones based on the transition probabilities tabulated by NIST (2003). This also implicitly confirms the validity of predicted transition probability ratios for the chosen 313.3, 312.2 and $311.6 \mathrm{~nm}$ O III spectral lines. Moreover, using the method (Djeniže \& Bukvić 2001) for the test of the self-absorption we can conclude that our investigated O III lines are not absorbed. Our line intensity ratio related to the $344.4 \mathrm{~nm}$ and $342.9 \mathrm{~nm}$ O III lines (Table 2) agree with values presented by Aller et al. (1966), obtained in astrophysical observations, and with theoretical predictions made by Froese Fischer (1994) and Kastner \& Bhatia (1990). Tolerable agreement was found (within the experimental accuracy and theoretical uncertainties) with the values presented by Liu \& Danziger (1993) (from observations of Bowen fluorescence mechanism and charge transfer in planetary nebulae); Aggarwal et al. (1997); Bhatia \& Kastner (1993). Line intensity ratios presented by Saraph \& Seaton (1980); NIST (2003) are about half the size of the others.

\subsection{Helium-oxygen plasmas}

The helium ions presence in the plasma verifies the role of the Bowen fluorescence mechanism. It is clear in the case of the $313.279 \mathrm{~nm}$ O III line which origins from the pumped $2 \mathrm{p} 3 \mathrm{~d}^{3} \mathrm{P}_{2}^{\mathrm{o}}$ energy level with $40.85 \mathrm{eV}$ excitation energy (NIST 2003). Namely, one can conclude that the $I(313.3) / I(311.6)$ line intensity ratio begins up with the increasing of the $\mathrm{He} / \mathrm{O}$ density ratio in plasma (see Figs. 2b,c,d). It turns out that the $311.6 \mathrm{~nm}$ O III line arises from the level $2 \mathrm{p} 3 \mathrm{~d}^{3} \mathrm{P}_{0}^{\mathrm{o}}$ with $40.87 \mathrm{eV}$ (NIST 2003) excitation energy, which is higher than the pumping He II photon energy. Therefore, this energy level remains without extra population processes due to the $\mathrm{He}$ II Ly $\mathrm{Ly}_{\alpha}$ photon absorption. The mentioned line intensity ratio depends on the 


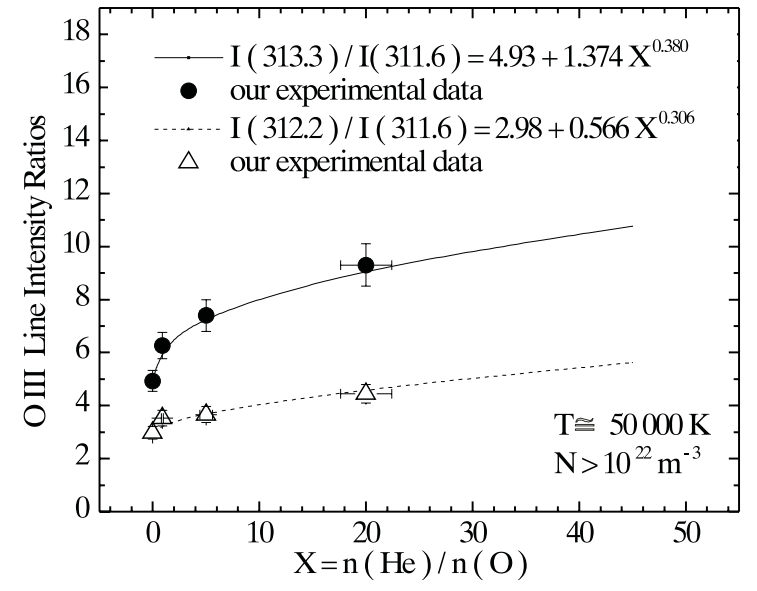

Fig. 3. O III line intensity ratios versus $X=n(\mathrm{He}) / n(\mathrm{O})$ density ratio at about $50000 \mathrm{~K}$ electron temperature and electron densities higher than $3 \times 10^{22} \mathrm{~m}^{-3}$. The line intensity ratios are obtained in the 10 th $\mu \mathrm{s}$ after the beginning of the discharge. The symbols $\bullet$ and $\Delta$ are related to the measured $I(313.3) / I(311.6)$ and $I(312.2) / I(311.6)$ O III line intensity ratios. The full and dashed lines denote their calculated values on the basis of the established Eqs. (2) and (3).

He II ion concentration and has its maximum at the moment when the He II $(468.6 \mathrm{~nm})$ line intensity reaches its maximum.

Similar behavior was found in case of the $I(312.2) / I(311.6)$ line intensity ratio (see Figs. 2b,c,d) which was not expected and observed up to now. It turns out that the $312.2 \mathrm{~nm} \mathrm{O}$ III line arises from the level $2 \mathrm{p} 3 \mathrm{~d}^{3} \mathrm{P}_{1}^{\mathrm{o}}$ with $40.86 \mathrm{eV}$ (NIST 2003) excitation energy. This level lies above the energy of the pumping photons and in the case of rare plasmas (with $N<10^{13} \mathrm{~m}^{-3}$ ) it is not included in the Bowen mechanism. We think that in the plasmas with $N>10^{22} \mathrm{~m}^{-3}$ electron density this level is broadened by the influence of an external micro-field created by electrons and ions (Griem 1974) and thus it is attainable for the pumping He II photons. In our experiment the electron density was higher than $2 \times 10^{22} \mathrm{~m}^{-3}$. So, we found an extra population tendency also in the case of the $2 \mathrm{p} 3 \mathrm{~d}^{3} \mathrm{P}_{1}^{\mathrm{o}} \mathrm{O}$ III level. The Bowen fluorescence mechanism has in this case a smaller role than in the case of the $2 \mathrm{p} 3 \mathrm{~d}^{3} \mathrm{P}_{2}^{\mathrm{o}}$ level (see also the $I(313.3) / I(312.2)$ line intensity ratios in Figs. 2b,c,d). On the basis of the found dependence of the line intensity ratios on the $X=n(\mathrm{He}) / n(\mathrm{O})$ density ratio (see Fig. 3) we have established the relationships between them.

They are:

$I(313.3) / I(311.6)=4.93+1.374 X^{0.380}$

and

$I(312.2) / I(311.6)=2.98+0.566 X^{0.306}$

obtained with high correlation factors of fitting (0.99).

Equations (2) and (3) can be used for calibration purposes in astrophysical and laboratory plasmas in the range of the cited plasma parameters.

In the case of the $I(344.4) / I(342.9)$ line intensity ratio we have found agreement with the value obtained in pure oxygen plasma (see Table 2) within our experimental accuracy of $\pm 8 \%$.
Taking into account that both the lines arise from the same energy level $\left(2 \mathrm{p} 3 \mathrm{~d}^{3} \mathrm{P}_{2}^{\mathrm{o}}\right)$ one can conclude that the presence of the Bowen mechanism does not influence their radiation probability ratio. Because this line intensity ratio does not depend on the helium presence in plasmas, it represents a convenient value in astrophysical plasma diagnostics.

\section{Conclusion}

We have found that in helium-oxygen plasmas with $N>$ $10^{22} \mathrm{~m}^{-3}$ and $T \sim 50000 \mathrm{~K}$, the $312.163 \mathrm{~nm} \mathrm{O}$ III line also shows a fluorescence tendency caused by the Bowen effect. We recommend the $I(313.379 \mathrm{~nm}) / I(311.567 \mathrm{~nm})$ O III line intensity ratio as a measure of the $\mathrm{He} / \mathrm{O}$ density ratio in plasmas with $N<10^{23} \mathrm{~m}^{-3}$ and $T<50000 \mathrm{~K}$. Our method is not sensitive to the presence of the helium ions and also to the possible self-absorption of the He II $\mathrm{P} \alpha$ line used offer for astrophysical plasma diagnostic purposes. We have also found that the $I(344.405 \mathrm{~nm}) / I(342.863 \mathrm{~nm})$ O III line intensity ratio, in the primary Bowen cascade, not dependent on the helium presence in plasma. On the basis of our results and those of other authors its proposed value is $5.94 \pm 14 \%$.

Acknowledgements. This work is a part of the project "Determination of the atomic parameters on the basis of the spectral line profiles" supported by the Ministry of Science, Technologies and Development of the Republic of Serbia. S. Djeniže is grateful to the Foundation “Arany János Közalapitvány” Budapest, Hungary.

\section{References}

Aggarwal, K. M., Hibbert, A., \& Keenan, F. P. 1997, ApJS, 108, 393 Aller, L. H., Kaler, J. B., \& Bowen, I. S. 1966, ApJ, 144, 291

Bhatia, A. K., \& Kastner, S. O. 1993, At. Data Nucl. Data Tables, 54, 133

Bowen, I. S. 1934, PASP, 46, 146

Djeniže, S., \& Bukvić, S. 2001, A\&A, 365, 252

Djeniže, S., Milosavljević, V., \& Dimitrijević, M. S. 2002a, A\&A, 382, 359

Djeniže, S., Srećković, A., Jelisavčić, M., \& Bukvić, S. 2002b, A\&A, 389, 1086

Djeniže, S., Dimitrijević, M. S., Srećković, A., \& Bukvić, S. 2002c, A\&A, 396, 331

Djeniže, S., Bukvić, S., Srećković, A., \& Kalezić, S. 2003, A\&A, 406, 759

Froese Fischer, C. 1994, Phys. Scr., 49, 51

Griem, H. R. 1964, Plasma Spectroscopy (New York: Mc. Graw Hill Inc.)

Griem, H. R. 1974, Spectral Line Broadening by Plasmas (New York: Acad. Press)

Harrington, J. P., Lutz, J. H., \& Seaton, M. J. 1981, MNRAS, 195P, $21 \mathrm{H}$

Kallman, T., \& McCray, R. 1980, ApJ, 242, 615

Kastner, S. O., \& Bhatia, A. K. 1990, ApJ, 362, 745

Liu, X.-W., \& Danziger, J. 1993, MNRAS, 261, 465

Milosavljević, V., \& Djeniže, S. 2002, A\&A, 393, 721

NIST 2003 - Atomic Spectra Data Base Lines http://physics.nist.gov

Rompe, R., \& Steenbeck, M. 1967, Ergebnisse der Plasmaphysik und der Gaselektronik, Band 1 (Berlin: Akademie Verlag)

Saraph, H. E., \& Seaton, M. J. 1980, MNRAS, 193, 617

Shrader, C. R., Singh, K. P., \& Barrett, P. 1997, ApJ, 486, 1006 